\title{
THE APRIL MEETING OF THE SAN FRANCISCO SECTION
}

The thirty-ninth regular meeting of the San Francisco Section of the American Mathematical Society was held at Stanford University on Saturday, April 8. Professor R. E. dllardice presided. The total attendance was twenty-one, including the following sixteen members of the Society:

Alderton, Allardice, Bernstein, Blichfeldt, Buck, Cajori, Daus, Haskell, Hoskins, Lehmer, Libby, W. A. Manning, Moreno, F. R. Morris, Noble, Pauline Sperry.

The Secretary of the Section was directed to send a letter of felicitation to the Chicago Section on the occasion of its twenty-fifth anniversary, and a letter of felicitation to Professor Scott on the occasion of the celebration held in her honor at Bryn Mawr College on April 18.

The dates of the next two regular meetings of the Section were fixed as October 21, 1922, University of California, and April 7, 1923, Stanford University.

Titles and abstracts of papers read at this meeting follow below. The papers of Professors Bell and Carpenter were read by title.

1. Dr. P. H. Daus: On the solution of the diophantine equation $x^{3}-D y^{3}= \pm 1$. Preliminary report.

The author finds solutions of the equation $X^{3}-D Y^{3}= \pm 1$ from the solutions of the pellian cubic $x^{3}+D y^{3}+D^{2} z^{3}$ $-3 D x y z=1$, and shows by means of ternary continued fractions that the first equation cannot have more than one non-trivial solution, and if it has a solution, it is obtainable from the fundamental solution of the pellian cubic.

2. Professor Florian Cajori: Recent symbolisms for decimal fractions.

The author describes the struggle for supremacy of the dot and the comma in different countries during the eighteenth and nineteenth centuries, as the decimal separatrix. During the past thirty-five years over half a dozen different decimal 
notations have appeared in print, viz., 2.5 (in the United States), $2 \cdot 5$ (in Great Britain), 2,5 (the common notation on the European Continent), 2’5, 2`5, 2,5 (three notations occasionally found in Spanish books), 2, ${ }_{5}, 2.5$ (two notations found in Scandinavia and Denmark).

\section{Professor Florian Cajori: Pricked letters and ultimate} ratios.

In the Philosophical Transactions (London), vol. 3, p. 763, N. Mercator used in 1668 the dot placed over the letter $I$ or over a number, to indicate an infinitesimal difference, similar to the use of the dot in $\dot{x}$ among certain English writers (after Newton in 1693 had used $\dot{x}$ in print to designate a fluxion or velocity) to denote infinitely small quantities. Mercator used in 1668 the Latin forms for "prime" and "ultimate little ratios"; Newton first used in print the phrase "prime and ultimate ratios" in 1687. It is possible that Newton adopted this terminology from Mercator, but his use of the dot in private papers antedates Mercator's use of it in print.

4. Professor B. A. Bernstein: The complete existential theory of Hurwitz's postulates for abelian groups and fields.

In a previous paper, the author determined those sets of Hurwitz's postulates for abelian groups and fields that are completely independent. In the present paper he obtains the complete existential theory of Hurwitz's remaining postulate-sets and thus establishes the complete existential theory of all of the sets. The complete paper will appear in this Bulletin.

5. Professor E. T. Bell: Periodicities in the theory of partitions.

Theta functions, not merely theta constants as customary, being used in the analysis of partitions, it is shown that Euler's $P(n)$ is connected with six new denumerants by a remarkable system of periodic relations. Two kinds of periodicity are involved: ordinary (as in analysis); numerical (periodicity of residues with respect to an integer modulus). Either or both can occur in one relation. The theory is closely connected with cyclotomy, Lucas' $u_{n}, v_{n}$, and the algebraic integers of a realm. The paper will appear in the Annals of Mathematics. 
6. Professor E. T. Bell: A revision of the Bernoullian and Eulerian functions.

Four functions of a single variable are taken as fundamental instead of the usual pairs of Raabe, Schlömilch, Worpitzky, Glaisher and others, and by these the theory is reduced by the symbolic method to a simple and symmetric form.

7. Professor E. T. Bell: Trigonometry and the numbers $B, E, G, R$ of Bernoulli, Euler, Genocchi, and Lucas.

All general relations between numbers $B, E, G, R$ of different ranks can be obtained briefly and systematically by Blissard's umbral calculus as an isomorph of trigonometry. A set of 22 such relations involving an arbitrary analytic function is given. The set is complete in that it contains at least one relation of each type. A relation is of type $r$ if it involves precisely $r$ of the symbols $B, E, G, R$.

8. Professor C. A. Noble: Retention of a salt solution in a tank of flowing water.

The author assumes that a salt solution of given strength is continuously admitted into a stream of water which flows uniformly through a system of $n$ tanks; that the contents of each tank is agitated; and that the salt diffuses instantaneously. He deduces the differential equation for the amount of salt remaining in the $n$th tank at the time $t$, and solves this equation.

9. Professor A. F. Carpenter: Generalization of a theorem on flecnode curves of ruled surfaces.

In this paper the author proves that a necessary and sufficient condition that the planes osculating the flecnode curve and the complex curve of a ruled surface at the four points in which they intersect any ruling form a harmonic pencil is the vanishing of the invariant

$$
\frac{p_{21}}{p_{12}} \Delta_{1}-\frac{p_{12}}{p_{21}} \Delta_{2} .
$$

This theorem generalizes certain results formerly obtained by the author (Transactions of THIS SocIETY, vol. 16 (1915), p. 509). The paper further determines the differential equations of the ruled surface generated by the axis of the pencil of osculating planes and shows that if the tangents to one branch of the flecnode curve of the given ruled surface 
cut out one branch of the flecnode curve of the second ruled surface, the same is true of the tangents to the other branch of the flecnode curve, that a corresponding situation holds for the respective complex curves, and finally that the two ruled surfaces are under these conditions space duals.

10. Professor Florian Cajori: The history of trigonometric notations.

The author describes trigonometric notations used by early writers, beginning with Thomas Finck, who in 1583 used contractions sin., tan., sec., sin.com., tan.com., sec.com.; he had very few followers in his time. In Great Britain, in 1618 and 1631, severe contractions like $s, t, s c o, t c o$ were introduced by Oughtred, Norwood and others for sine, tangent, cosine, cotangent, but seldom did any two authors or even any one author always adhere to exactly the same set of symbols. About 1760 these severe contractions were abandoned in England. There and on the continent abbreviations of three or four letters (five letters for cosec) were usually preferred, but no unanimity exists even today. The origin is traced of notations for the inverse functions (arc sin (Euler), $\sin ^{-1} x$ (J. F. W. Herschel), $\frac{1}{\sin } x$ (M. Ohm)), also for hyperbolic functions ( $\sinh$ (V. Riccati and J. H. Lambert), Sin (C. Gudermann)) and for parabolic functions (J. Booth).

11. Dr. F. R. Morris: Arrangement of interest tables for Liberty Bonds.

The usual plan of interest tables for bonds is to place the time until maturity and rate of income in the two margins and the value of the bond in the body of the table. However, it is often desirable to read the rate directly. In the case of Liberty Bonds, compact tables may be constructed such that rate may be read if the market price on a given date is known. With time and rate as arguments the computation is direct; with time and value as arguments the computation is indirect and rather difficult.

12. Professor H. F. Blichfeldt: Notes on diophantine approximations. Preliminary report.

The following is a well known theorem: $n$ irrational numbers $\alpha_{1}, \cdots, \alpha_{n}$ being given, as well as a positive number $\epsilon$ ar- 
bitrarily small, then a set of $n$ rational fractions $x_{1} / y, \cdots, x_{n} / y$ can be found such that $\left|\alpha_{i}-x_{i} / y\right|<\epsilon$ and also $<\delta / y^{1+1 / n}$ $(i=1,2, \cdots, n)$, where $\delta$ is a fixed number depending upon $n$ only (cf. Transactions of this Society, vol. 15 (1914), pp. 234-5; L. E. Dickson, History of the Theory of Numbers, vol. 2, p. $95 \mathrm{ff}$.). A. Hurwitz has proved (loc. cit.) that $\delta=1 / \sqrt{5}$ for $n=2$; and H. F. Blichfeldt that $\delta \geqq 1 / \sqrt[4]{23}$ for $n=3$ (proof not yet published). In Mathematische Annalen, vol. 83 (1921), p. $77 \mathrm{ff}$., there is a proof by $\mathrm{O}$. Perron that $\delta>(1 / n)[0.35 /(n+1)]^{n}$ approximately. The present writer, using Perron's method with refinements based on the principles of the geometry of numbers, obtains the somewhat better result $\delta>(N / \sqrt{n})\left(2 /{\sqrt{n)^{n}}}^{n}\right.$ approximately, where $N$ is a fixed number.

\section{B. A. Bernstein, Secretary of the Section.}

\section{THE EASTER MEETING OF THE SOCIETY}

The two hundred twenty-second regular meeting of the American Mathematical Society, being the seventeenth regular Western meeting, and the forty-ninth regular meeting of the Chicago Section, was held at the University of Chicago on Friday and Saturday, April 14 and 15, 1922, in honor of the twenty-fifth anniversary of the Chicago Section. The attendance at these meetings was approximately one hundred fifty, among whom were the following one hundred four members of the Society:

E. S. Allen, F. E. Allen, Baker, Beckwith, Bliss, Blumberg, Bradshaw, Brahana, Brooke, Bussey, C. C. Camp, Carmichael, Chapman, Chittenden, Coble, Crathorne, H. B. Curtis, Curtiss, Dalaker, H. T. Davis, Denton, Dickson, Doll, Dowling, Dresden, Escott, Everett, Eversull, FeldsteinTartakovsky, Feltges, Fields, Fry, Gibbens, Glenn, Gouwens, Green, W. L. Hart, M. G. Haseman, E. R. Hedrick, Hildebrandt, Hoar, Hodge, Ingraham, Dunham Jackson, Kinney, Lane, Lennes, Logsdon, Lunn, McGaw, M. M. McKelvey, J. V. McKelvey, N. B. MacLean, MacMillan, March, Marshall, T. E. Mason, Meacham, B. I. Miller, E. B. Miller, G. A. Miller, Miser, C. N. Moore, E. H. Moore, E. J. Moulton, F. R. Moulton, A. L. Nelson, Newson, Olson, C. I. Palmer, Pitcher, J. F. Reilly, R. G. D. Richardson, Rider, H. L. Rietz, Risley, Roever, Roman, Schottenfels, Schweitzer, Shaw, W. G. Simon, Skinner, Slaught, Edwin R. Smith, Stecker, Steimley, Stouffer, E. L. Thompson, B. M. Turner, J. S. Turner, 